\title{
Factores asociados a alopecia no cicatricial en mujeres adultas, estudio transversal entre enero y diciembre 2013
}

\section{Associated factors with nonscarring alopecia in adult women, cross- sectional study carried out from January to December 2013 \\ Fatores associados à alopecia não cicatricial em mulheres adultas, estudo transversal entre janeiro e dezembro de 2013}

Leonor Inés Cifuentes-Tang, MD., Esp.*

Juan Carlos Uribe-Caputi, MD., MSc.**

\section{Resumen}

Introducción. Alopecia significa caída patológica del pelo, de cualquier tipo y en cualquier lugar de la superficie cutánea. Se divide en dos grandes grupos: cicatricial y no cicatricial. Esta última, a pesar de ser una patología prevalente, no ha sido documentada en la población latinoamericana. Objetivo. Establecer la prevalencia y las variables asociadas a la alopecia, de interés en la población de estudio. Métodología. Estudio observacional de tipo analítico, realizado entre enero y diciembre de 2013. Se revisaron 169 historias clínicas de mujeres mayores de 18 años con alopecia no cicatricial. El análisis estadístico se realizó con Epi Info versión 7. Resultados. La edad promedio para el total de los casos fue de 38.5 años. La prevalencia estimada para las alopecias no cicatriciales fue de 12.1\%; el 35.5\% (IC 95\% 28.31-43.22) fue diagnosticado como pérdida capilar de patrón femenino, el
19.53\% (IC 95\% 13.84-26.31) como alopecia mixta y alopecia areata, seguida del efluvium telógeno crónico con un $17.16 \%$ (IC 95\% 11.8-23.7). La mayoría tenía algún antecedente médico de importancia, principalmente hipotiroidismo con 20.47\% (IC 95\% 13.8-28.5). De las pacientes evaluadas, el valor promedio de ferritina, tirotropina y hemoglobina fue $76.65 \mathrm{mcg} / \mathrm{L}, 3.08 \mathrm{ml} \mathrm{U} / \mathrm{L}$ y 13.81 gramos/dl respectivamente. Se encontró asociación positiva, entre tipo de alopecia y ferritina $(\mathrm{p}<0.0001)$. Conclusiones. El presente estudio concluye que la ferritina sérica disminuida está relacionada con la fisiopatología del efluvium telógeno, lo que no ocurre en los trastornos tiroideos. Se requieren más estudios para establecer las variables asociadas en Colombia. [CifuentesTang LI, Uribe-Caputi JC. Factores asociados a alopecia no cicatricial en mujeres adultas, estudio transversal entre enero y diciembre 2013. MedUNAB. 2018;21(1):59-66. doi: 10.29375/01237047.2677].

\footnotetext{
* Médica, especialista en Epidemiología, Residente de Dermatología, Universidad Libre seccional Cali, Cali, Valle del Cauca, Colombia.

** Médico, magíster en Epidemiología, Facultad de Ciencias de la Salud, Universidad Autónoma de Bucaramanga, Bucaramanga, Santander, Colombia Correspondencia: Leonor Inés Cifuentes-Tang. Carrera 36 B No. 5B 3-29, Cali, Valle del Cauca, Colombia. Teléfono: +57 3186934010. E-mail: lcifuentes10@unab.edu.co.
} 
Palabras clave: Alopecia; Anemia; Deficiencia de Hierro; Ferritinas; Prevalencia; Colombia.

\section{Abstract}

Introduction. Alopecia refers to the pathological loss of hair in any form and within any place of the skin surface. This medical condition is divided into two large groups: cicatricial and nonscarring alopecia. Although the second type of Alopecia is a prevalent pathology, it has not been recorded among LatinAmerican population. Objective. To establish the prevalence and variables associated with alopecia that are relevant to the study population. Methodology. Analytical observational study that was carried out from January to December of 2013. This one reviewed 169 medical records of women over the age of 18 that had nonscarring alopecia. The statistical analysis was made using EPI Info version 7. Results. The average age of the reviewed medical cases for this study was 38.5 and the estimated prevalence for nonscarring alopecia was $12.1 \%$. $35.5 \%$ (IC 95\% 28.31-43.22) was diagnosed as female pattern of hair loss; $19.53 \%$ (IC 95\% 13.84-26.31) was diagnosed as mixed alopecia and alopecia areata, followed by chronic telogen effluvium with $17.16 \%$ (IC 95\% 11.8-23.7). The great majority of patients had significant medical histories, mostly those associated with hypothyroidism with a $20.47 \%$ (IC 95\% 13.8-28.5). Among the patients that were studied, the average rate for ferritin, thyrotropin and hemoglobin was $76.65 \mathrm{mcg} / \mathrm{L}$, $3.08 \mathrm{ml} \mathrm{U} / \mathrm{L}$ and 13.81 grams/dl respectively. The study showed a positive association between the alopecia type and ferritin rates $(p<0.0001)$. Conclusions. This study concludes that reduced rates of serum ferritin are related with telogen effluvium pathophysiology and that this condition does not occur in thyroid disorders. More studies are required in order to stablish variables associated with Colombia. [Cifuentes-Tang LI, Uribe-Caputi JC. Associated factors with nonscarring alopecia in adult women, cross-sectional study carried out from January to December 2013. MedUNAB. 2018;21(1):5966.doi: 10.29375/01237047.2677].

Keywords: Alopecia; Anemia; Iron Deficiency; Ferritins; Prevalence; Colombia.

\section{Resumo}

Introdução. Alopecia significa queda de cabelo patológica, de qualquer tipo e em qualquer lugar na superficie da pele. Pode ser classificada em dois grandes grupos: cicatricial e não cicatricial. Este último, apesar de ser uma patologia prevalente, não foi documentado ainda na população latino-americana. Objetivo. Estabelecer a prevalência e as variáveis associadas à alopecia, de interesse na população estudada. Metodologia. Estudo observacional do tipo analítico, feito entre janeiro e dezembro de 2013. Foram revisados 169 prontuários de mulheres com mais de 18 anos com alopecia não cicatricial. A análise estatística foi realizada com o software Epi Info versão 7. Resultados. A idade média para o total de casos foi de 38,5 anos. A prevalência estimada para alopecias não cicatriciais foi de $12.1 \%$; 35.5\% (IC 95\% 28.31- 43.22) foi diagnosticada como perda capilar do padrão feminino, $19.53 \%$ (IC 95\% 13.84-26.31) como alopecia mista e alopecia areata, seguido por eflúvio telógeno crônico com 17.16\% (IC 95\% 11.8- 23.7). A maioria apresentava algúm histórico médico importante, principalmente hipotireoidismo com $20.47 \%$ (IC 95\% 13.8-28.5). Das pacientes avaliadas, o valor médio de ferritina, tirotropina e hemoglobina foi $76.65 \mathrm{mcg} / \mathrm{L}, 3.08$ $\mathrm{ml} \mathrm{U/L} \mathrm{e} 13.81$ gramas/dl respectivamente. Uma associação positiva foi encontrada entre o tipo de alopecia e ferritina $(p<0.0001)$. Conclusões. O presente estudo conclui que a diminuição da ferritina sérica está relacionada à fisiopatologia do eflúvio telógeno, o que não ocorre nos distúrbios da tireoide. Mais estudos são necessários para estabelecer as variáveis associadas na Colômbia. [Cifuentes-Tang LI, UribeCaputi JC. Fatores associados à alopecia não cicatricial em mulheres adultas, estudo transversal entre janeiro e dezembro de 2013. MedUNAB. 2018;21(1):59-66. doi: 10.29375/01237047.2677].

Palavras-chave: Alopecia; Anemia; Deficiência de Ferro; Ferritinas; Prevalência; Colômbia.

\section{Introducción}

Alopecia significa caída patológica del pelo, de cualquier tipo y en cualquier lugar de la superficie cutánea (1). Raymond J. Adrien Sabouraud acuñó el término alopecia y lo incluyó como diagnóstico dermatológico, ampliando su aplicación a cualquiertipo de caída patológica del pelo, concepto que se mantiene actualmente (1). Las alopecias se dividen en dos grandes grupos: las cicatriciales y las no cicatriciales. En las alopecias cicatriciales el folículo piloso se destruye y la pérdida del cabello es irreversible. En las no cicatriciales, el folículo persiste y, por lo tanto, puede haber regeneración del pelo (2). Para entenderla, es necesario describir en forma breve el ciclo normal del pelo. Cada folículo piloso pasa continuamente por tres fases: anágena (fase de crecimiento) que dura de tres a seis años; catágena (fase de involución), dura unas tres semanas; y telógena (fase de reposo), con una duración de tres meses (3).

La pérdida capilar de patrón femenino (FPHL, por sus siglas en inglés) se define como la miniaturización progresiva del folículo piloso, en mujeres predispuestas genéticamente (4), con una conversión de los folículos 
terminales a folículos similares al vello, el cual tiene un ciclo más corto (anágena reducida) produciendo cabellos cortos, finos y no pigmentados $(4,5)$. El efluvium telógeno (ET) fue descrito por primera vez por Kligman en 1961 (6) y resulta de la transición síncrona de los folículos capilares desde la etapa de crecimiento del ciclo del pelo (anágena) hasta la etapa de reposo del ciclo del pelo (telógeno) $(7,8)$. Los eventos desencadenantes comunes incluyen el parto, la fiebre y algunos medicamentos, aunque, a menudo, dichos factores no son discernibles (9). ET que dura más de 6 meses se conoce como efluvium telógeno crónico (ETC) (10) y a menudo queda enmascarado en el seno de la FPHL (1). Las causas conocidas de ET, tales como alteración de la función tiroidea, del hierro o proteínas, deben ser excluidas antes de poder hacer el diagnóstico de ETC (10).

La alopecia areata (AA) es un proceso autoinmune, asociado con un infiltrado linfocítico que rodea los bulbos capilares $(11,12)$. Se caracteriza por la presencia de una o varias placas circunscritas sin pelo, sobre piel normal, y suele ser asintomática (1). Al examen físico, se observan pelos en punto de exclamación; generalmente presenta remisión espontánea $\mathrm{y}$, en ocasiones, recurrencias (1). Norwood y colegas reportaron una prevalencia del 19\% para FPHL, en una serie de 1,000 mujeres caucásicas para el año 2001. En un estudio británico de 377 pacientes, reportaron 38\% en mujeres mayores de 70 años y un estudio coreano reportó una prevalencia del 5.6\% independientemente de la edad. Sin embargo, no se encontraron datos epidemiológicos sobre esta patología con referencia a mujeres africanas y de países latinoamericanos (13). Estados Unidos reporta una incidencia del $12 \%$ en mujeres de 30 años y del 30 al $40 \%$ en mujeres de 60 a 69 años de edad (4). Un estudio sobre FPHL, realizado en la ciudad de Bogotá por la Universidad del Rosario y Universidad CES en mayo de 2015, reporta una prevalencia del $20.84 \%$ en una serie de 99 mujeres que estuvieron en consulta dermatológica (14). El objetivo del presente estudio es establecer la prevalencia y las variables asociadas de la alopecia no cicatricial que, aunque se considera una patología frecuente en la población femenina, no ha sido documentada en la población latinoamericana. Por ello, se quiere establecer su prevalencia a nivel local, así como sus comorbilidades.

\section{Metodología}

Estudio transversal observacional de tipo analítico, realizado entre enero y diciembre de 2013. Se revisaron
169 historias clínicas de mujeres pertenecientes al régimen contributivo, entre 18-70 años de edad, con diagnóstico de alopecia no cicatricial, obtenida de la base de datos de la consulta externa de Salud Total EPS (Entidad Promotora de Salud), de la ciudad de Manizales.

Se incluyeron mujeres con caída del cabello entre 1870 años de edad, clasificadas dentro de las alopecias no cicatriciales y a quienes fueron solicitados los paraclínicos de interés (ferritina, hemoglobina y hematocrito, serología, hormona estimulante de la tiroides). Se excluyeron: mujeres con enfermedades autoinmunes del tejido conectivo que afectan la piel cabelluda (lupus discoide o liquen pilaris); alopecias secundarias a inflamaciones superficiales de piel cabelluda tales como tiña cápitis, dermatitis seborreica o psoriasis; alopecia en una zona corporal distinta a escalpo; y alopecia de tipo psicógena (tricotilomanía).

La información recolectada fue ingresada en una base de datos, previamente diseñada en formato Microsoft Excel, y analizada con Epi Info versión 7. El signo pull test se utiliza para evaluar la resistencia o fragilidad del tallo capilar. Se toma un mechón de 40-60 cabellos entre los dedos pulgar e índice y se aplica una tracción suave y firme del cuero cabelludo, categorizándose como presente o ausente, según el caso. La ferritina se categorizó como normal si mayor de 70 microgramos/ litro; baja entre 35-70 microgramos/litro; y ferritina muy baja: menor de 35 microgramos/litro. La tirotropina (TSH) se categorizó como normal: $0.3-4.12 \mathrm{mIU} / \mathrm{L}$; TSH: 4.5-10 mIU/L (hipotiroidismo subclínico); TSH $>10$ (hipotiroidismo franco); y TSH: 0.1-0.45 mIU/L (hipertiroidismo), siguiendo al Journal of the American Medical Association (JAMA). La hemoglobina ( $\mathrm{Hb})$ se categorizó como normal: 12.1 a 15.8 gramos/dl; baja: $<12.0$ gramos/dl; y alta $>18.0$ gramos/dl, según The American Society of Hematology.

En el análisis estadístico, los datos numéricos que tuvieron un comportamiento normal se presentan en promedios y desviaciones estándar (DE) y los no normales en mediana y rangos. Las variables cualitativas se expresaron como ausencia o presencia del evento y en frecuencias y porcentajes. Las asociaciones se establecieron mediante pruebas de chicuadrado. El protocolo de investigación fue aprobado por el Comité de Ética para la Investigación de la Universidad Autónoma de Bucaramanga; también fue avalado por el Comité de Ética de Salud Total EPS. 


\section{Resultados}

La prevalencia estimada para la alopecia no cicatricial fue del $12.1 \%$, con un promedio de edad para el total de la muestra de 38.5 años, con una DE 12.13 (Tabla 1). Con referencia al curso de la enfermedad, el tiempo promedio fue 2.9 años (rango entre un mes y 27 años), con una DE 4.04 .

El valor promedio de ferritina fue $76.65 \mathrm{mcg} / \mathrm{L}$ (rango 3.9-401.8 mcg/L), con una DE 68.22; con una ferritina normal en el 37.17\% (IC 95\% 28.2-46.7); baja $30.97 \%$ (IC 95\% 22.6-40.3); y muy baja $31.86 \%$ (IC 95\% 23.4-41.2). El valor promedio del TSH fue $3.08 \mathrm{ml} \mathrm{U} / \mathrm{L}$ (rango 0.13-12.12 ml U/L) con una DE 1.95 , con un TSH normal en un $77.17 \%$ (IC 95\% 68.8-
84.1), hipotiroidismo en $20.47 \%$ (IC 95\% 13.8-28.5) e hipertiroidismo en $2.36 \%$ (IC 95\% 0.4-6.7). El valor promedio de la $\mathrm{Hb}$ fue 13.81 gramos/dl (rango 10.5215.9 gramos/dl) con una DE 1.02, con una Hb normal en un 91.53\% (IC 95\% 84.9-95.8), baja 5.93\% (IC $95 \% 2.4-11.8$ ) y alta en un $2.54 \%$ (IC $95 \% 0.5-7.2$ ).

Con relación al signo pull test, el 76.92\% (IC 95\% 69.8-83) fue negativo en FPHL y AA, el $10.06 \%$ (IC 95\% 5.9-15.6) fue positivo en ETC y en alopecia mixta (FPHL más ETC) (Tabla 2). En la Tabla 3, se muestra el análisis bivariado de la población estudiada.

Tabla 1. Variables sociodemográficas

\begin{tabular}{|c|c|c|c|}
\hline Variables sociodemográficas & $\mathrm{n}$ & $\%$ & IC 95\% \\
\hline \multicolumn{4}{|l|}{ Procedencia } \\
\hline Manizales & 118 & 69.82 & $62.3-76.6$ \\
\hline Villamaría & 33 & 19.53 & $13.8-26.3$ \\
\hline Chinchiná & 18 & 10.65 & 6.4-16.3 \\
\hline \multicolumn{4}{|l|}{ Escolaridad } \\
\hline Primaria & 20 & 11.83 & 7.3-17.6 \\
\hline Secundaria & 102 & 60.36 & $52.5-67.7$ \\
\hline Técnica & 23 & 13.61 & 8.8-19.7 \\
\hline Universitaria & 24 & 14.2 & 9.3-20.3 \\
\hline \multicolumn{4}{|l|}{ Ocupación } \\
\hline Hogar & 59 & 34.91 & $27.7-42.6$ \\
\hline Ventas & 24 & 14.2 & 9.3-20.3 \\
\hline Aseo & 14 & 8.28 & 4.6-13.5 \\
\hline \multicolumn{4}{|l|}{ Estado civil } \\
\hline Casada & 55 & 32.5 & $25.5-40.1$ \\
\hline Unión libre & 48 & 28.4 & 21.7-35.8 \\
\hline Soltera & 47 & 27.81 & 21.2-35.2 \\
\hline Separada & 13 & 7.49 & $4.1-12.7$ \\
\hline Viuda & 6 & 3.55 & $1.3-7.5$ \\
\hline
\end{tabular}

Fuente: Elaboración propia de los autores 
Tabla 2. Variables de caracterización clínica

\begin{tabular}{cccc}
\hline $\begin{array}{c}\text { Caracterización clínica } \\
\text { Tipo de alopecia }\end{array}$ & $\mathbf{n}$ & $\%$ & IC 95\% \\
FPHL & 60 & 35.5 & $28.3-43.2$ \\
AA & 33 & 19.53 & $13.8-26.3$ \\
A. mixta & 33 & 19.53 & $13.8-26.3$ \\
ETC & 29 & 17.16 & $11.8-23.7$ \\
No clasificadas & 14 & 8.28 & $4.6-3.5$ \\
Antecedentes patológicos & & & \\
Hipotiroidismo & 32 & 18.93 & $13.3-25.6$ \\
Gastritis & 28 & 16.57 & $11.3-23$ \\
Negaron & 27 & 15.98 & $10.8-22.3$ \\
Hipertensión & 9 & 5.33 & $2.4-9.8$ \\
Diabetes & 3 & 1.78 & $0.3-5.1$ \\
Antecedentes de alopecia & & & \\
Sin registro & 69 & 40.83 & $33.3-48.6$ \\
No & 59 & 34.91 & $27.7-42.6$ \\
Sí & 41 & 24.26 & $18.0-31.4$
\end{tabular}

Signo pull test

$\begin{array}{cccl}\text { No } & 130 & 76.92 & 69.8-83 \\ \text { Sí } & 17 & 10.06 & 5.9-15.6 \\ \text { Sin registro } & 22 & 13.02 & 8.3-19\end{array}$

Manejos cosméticos

$\begin{array}{crrr}\text { No } & 11 & 6.51 & 3.2-11.3 \\ \text { Sí } & 82 & 48.52 & 40.7-56.3 \\ \text { Sin registro } & 76 & 44.97 & 37.3-52.8\end{array}$

Remisión dermatología

\begin{tabular}{cccc} 
No & 72 & 42.6 & $35.0-50.4$ \\
Sí & 97 & 57.39 & $49.5-64$ \\
\hline
\end{tabular}

*FPHL: pérdida capilar de patrón femenino, AA: Alopecia areata, ETC: efluvium telógeno crónico, IC: intervalo de confianza. Fuente: Elaboración propia de los autores 
Tabla 3. Asociaciones clínicas y paraclínicas frente al desenlace

\begin{tabular}{|c|c|c|c|}
\hline Tipo de alopecia & & $\%$ & p Valor \\
\hline \multicolumn{4}{|l|}{ Duración de la alopecia } \\
\hline \multirow{2}{*}{ Menor de 1 año } & $\mathrm{AA}$ & 66.67 & $<0.0001$ \\
\hline & ETC & 58.62 & $<0.0001$ \\
\hline \multirow[t]{2}{*}{ Entre 1 y 5 años } & FPHL & 60.00 & $<0.0001$ \\
\hline & A. mixta & 57.58 & $<0.0001$ \\
\hline \multicolumn{4}{|l|}{ Ferritina } \\
\hline \multirow[t]{2}{*}{ Muy bajo } & A. mixta & 51.52 & $<0.0001$ \\
\hline & ETC & 48.15 & $<0.0001$ \\
\hline \multirow[t]{2}{*}{ Bajo } & A. mixta & 48.48 & $<0.0001$ \\
\hline & ETC & 48.15 & $<0.0001$ \\
\hline Normal & FPHL & 83.33 & $<0.0001$ \\
\hline \multicolumn{4}{|l|}{ Signo pull test } \\
\hline \multirow[t]{3}{*}{ Negativo } & FPHL & 93.33 & $<0.0001$ \\
\hline & A. mixta & 87.88 & $<0.0001$ \\
\hline & $\mathrm{AA}$ & 69.70 & $<0.0001$ \\
\hline Positivo & ETC & 24.14 & $<0.0001$ \\
\hline \multirow{3}{*}{ Manejos cosméticos } & FPHL & 65.00 & $<0.0001$ \\
\hline & A. Mixta & 60.61 & $<0.0001$ \\
\hline & ETC & 48.28 & $<0.0001$ \\
\hline Sin manejo cosmético & A. mixta & 18.18 & $<0.0001$ \\
\hline \\
\hline \multirow{3}{*}{ Normal } & A. mixta & 81.81 & $<0.49$ \\
\hline & ETC & 76.92 & $<0.49$ \\
\hline & FPHL & 75.47 & $<0.49$ \\
\hline \multirow{3}{*}{ Hipotiroidismo } & FPHL & 22.64 & $<0.49$ \\
\hline & ETC & 19.23 & $<0.49$ \\
\hline & A. Mixta & 18.18 & $<0.49$ \\
\hline \multicolumn{4}{|l|}{ Hemoglobina } \\
\hline \multirow[t]{3}{*}{ Normal } & ETC & 96.15 & $<0.43$ \\
\hline & FPHL & 93.88 & $<0.43$ \\
\hline & A. Mixta & 86.87 & $<0.43$ \\
\hline \multirow[t]{2}{*}{ Baja } & A. mixta & 6.67 & $<0.43$ \\
\hline & FPHL & 4.08 & $<0.43$ \\
\hline \multicolumn{4}{|l|}{ Antecedente familiar } \\
\hline \multirow[t]{3}{*}{ Sí } & $\mathrm{FPHL}$ & 38.33 & $<0.0001$ \\
\hline & A. Mixta & 33.33 & $<0.0001$ \\
\hline & ETC & 20.69 & $<0.0001$ \\
\hline \multirow[t]{3}{*}{ No } & A. Mixta & 48.48 & $<0.0001$ \\
\hline & ETC & 44.83 & $<0.0001$ \\
\hline & FPHL & 41.67 & $<0.0001$ \\
\hline \multicolumn{4}{|l|}{ Remisión Dermatología } \\
\hline \multirow{3}{*}{ Sí } & A. mixta & 72.73 & $<0.003$ \\
\hline & AA & 66.67 & $<0.003$ \\
\hline & FPHL & 58.53 & $<0.003$ \\
\hline \multirow[t]{2}{*}{ No } & No clasificadas & 85.71 & $<0.003$ \\
\hline & ETC & 51.72 & $<0.003$ \\
\hline \multicolumn{4}{|l|}{ Ferritina } \\
\hline Baja (Hb normal) & & 93.94 & $<0.13$ \\
\hline Muy Baja (Hb normal) & & 83.33 & $<0.13$ \\
\hline
\end{tabular}

*AA: alopecia areata, ETC: efluvium telógeno crónico, FPHL: pérdida capilar de patrón femenino, Hb: hemoglobina. Fuente: Elaboración propia de los autores 


\section{Discusión}

La prevalencia estimada por este estudio para el periodo 2013 fue de $12.1 \%$. Se puede comparar tanto con la descrita por Albarán y colegas, que reportaron una prevalencia de $20.84 \%$ en mujeres con FPHL en la ciudad de Bogotá, como con la presentada en mujeres Caucásicas por Norwood y colegas, que evidenciaron una prevalencia del 19\%. El análisis de los resultados evidenció la FPHL como la alopecia de mayor frecuencia, concordando con lo descrito por Werner y colegas. En segundo lugar se posicionaron las alopecias mixta y areata, situación que difiere de lo señalado por los mencionados autores, quienes describieron el efluvium telógeno como la segunda en frecuencia (15). Así, se confirmó de forma parcial en la hipótesis de trabajo.

Kantor y colegas señalaron que los 40 años son una edad promedio de las alopecias no cicatriciales (16), hallazgo similar a lo encontrado en el presente estudio. Werner y colegas describieron, al igual que el presente trabajo, que la herencia en alopecia androgenética es variable (15). Se encontraron como comorbilidades asociadas: hipotiroidismo, hipertensión arterial y diabetes; entidades similares a las descritas en el estudio de Vujovic. Estas enfermedades aumentan el riesgo cardiovascular en aquellas mujeres con FPHL temprana (13). Sin embargo, se requieren más estudios.

Según Vujovic y colegas, las pacientes con FPHL pueden tener pull test positivo durante la fase activa en el área afectada. Un pull test positivo requiere siempre investigación para excluir efluvium telógeno (13). Las pacientes de este estudio tuvieron categóricamente pull test negativo $(\mathrm{p}<0.0001)$. Según Burman y colegas la disfunción tiroidea es responsable de distintas manifestaciones dermatológicas, entre ellas la alopecia, encontrándose tanto en hipertiroidismo como en hipotiroidismo (17). Freinkel refiere que la alopecia en hipotiroidismo es adquirida, alargando la fase telógena de los folículos pilosos (18). El presente estudio mostró diferencias, al no encontrar significancia estadística entre los tipos de alopecia y TSH ( $<$ 0.49). Hallazgo similar fue reportado por Sinclair, apoyado por Moeinvaziri, quien no encontró una diferencia significativa entre los valores de TSH, T4 libre o tiroxina y la velocidad de sedimentación en mujeres con y sin pérdida capilar telógena (19).

Estudios observacionales han sugerido que la alopecia en mujeres puede asociarse a una disminución de las reservas de hierro corporal (20-22). Algunos estudios han sugerido que la disminución de las reservas de hierro en el cuerpo (medida por la ferritina sérica) puede asociarse con ET (22). Dos estudios observacionales evaluaron la asociación entre niveles reducidos de ferritina y AA, llegando a conclusiones opuestas. Sin embargo, ambos estudios estaban limitados por su metodología, que se basó en normas publicadas para ferritina y hemoglobina extraídas de laboratorios y poblaciones diferentes $(23,24)$. En la literatura revisada no se encontró un estudio que haya utilizado metodologías epidemiológicas estándar para evaluar la relación entre la alopecia en las mujeres y la disminución de las reservas de hierro.

Como una limitación, se encontró que, al ser un estudio de prevalencia, no se puede demostrar causalidad entre los trastornos tiroideos y las variaciones encontradas con los niveles de ferritina y hemoglobina, frente al desenlace. Esto da espacio para un futuro estudio, cuyo diseño y metodología se enmarquen en un diseño con seguimiento. Además, al ser el presente estudio de tipo retrospectivo, está sujeto a sesgos, que se minimizaron teniendo un adecuado tamaño de muestra y aplicando criterios de inclusión y exclusión. Se encontró como falencia el formato de recolección (historia clínica), ya que su diseño está enfocado para fines distintos a los de un estudio de investigación. Se observó que la calidad de los datos no era adecuada e, incluso, había ausencia de algunos de ellos.

\section{Conclusiones}

La ferritina sérica disminuida está relacionada con la fisiopatología del ET, no ocurriendo así con los trastornos tiroideos. A pesar de la prevalencia de la alopecia no cicatricial, pocos estudios epidemiológicos se han realizado para establecer la etiología y los factores de riesgo de este desorden, por lo que se recomienda un estudio con mayor tamaño de muestra, con el fin de caracterizar la prevalencia y las comorbilidades asociadas en la población colombiana.

\section{Consideraciones éticas}

Protección de personas y animales. Los autores declaran que en esta investigación no se han realizado experimentos en seres humanos ni en animales.

Confidencialidad de los datos. Los autores declaran que han seguido los protocolos de su centro de trabajo sobre publicación de datos de pacientes. 
Derecho a la privacidad y consentimiento informado. Los autores declaran que en este artículo no aparecen datos de pacientes.

\section{Conflicto de intereses}

Los autores declaran no detener conflicto de interés.

\section{Agradecimientos}

A Salud Total EPS por facilitar la información.

\section{Financiación}

Este artículo fue financiado por los autores.

\section{Referencias}

1. Guerra A. La alopecia de la mujer. 5 ed. Madrid: Editorial Raíz; 2009.

2. Falabella R, Escobar C, Giraldo N. Dermatología Fundamentos de Medicina. 5 ed. Medellín, Colombia: Corporación para investigaciones biológicas; 2002.

3. Thiedke CC. Alopecia in Women. Am Fam Physician. 2003; 67(5):1007-14.

4. Herskovitz I, Tosti A. Female pattern hair loss. Int J Endocrinol Metab. 2013;11(4):e9860. doi: 10.5812/ ijem.9860

5. Levy LL, Emer JJ. Female pattern alopecia: current perspectives. Int J Womens Health. 2013;5:541-56. doi: 10.2147/IJWH.S49337.

6. Malkud S. Telogen Effluvium: A Review. J Clin Diagn Res. 2015;9(9):WE01-03. doi: 10.7860/ JCDR/2015/15219.6492.

7. Headington JT. Telogen effluvium: New concepts and review. Arch Dermatol. 1993;129(3):356-63. doi: 10.1001/archderm.1993.01680240096017.

8. Harrison S, Sinclair R. Telogen Effluvium. Clin Exp Dermatol. 2002; 27(5):389-5. doi: 10.1046/j.13652230.2002.01080.x.

9. Paus R, Cotsarelis G. The biology of hair follicles. N Engl J Med. 1999;341(7):491-7. doi: 10.1056/ NEJM199908123410706.

10. Whiting DA. Chronic telogen effluvium: increased scalp hair shedding in middle aged women. J Am Acad Dermatol. 1996;35(6):899-906.

11. GilharA, Ullmann Y,Berkutzki T,Assy B, Kalish RS. Autoimmune hair loss (alopecia areata) transferred by T lymphocytes to human scalp explants on Scid mice. J Clin Invest. 1998;101(1):62-7. doi: 10.1172/ JCI551.

12. Bertolino AP. Alopecia areata. A clinical overview. Postgrad Med. 2000; 107(7):81-90. doi: 10.3810/ pgm.2000.06.1111.

13. Vujovic A, Del marmol V. The female pattern hair loss: review of etiopathogenesis and diagnosis. Biomed Res Int. 2014;2014:767628. doi: 10.1155/2014/767628.

14. Albarán C, Alejandro L, Chaparro A. Estudio descriptivo sobre la pérdida de cabello de patrón femenino en una consulta dermatológica general [Tesis]. Bogotá: Universidad del Rosario y CES; 2015. 75 p.

15. Werner B, Mulinari-Brenner F. Clinical and histological challenge in the differential diagnosis of diffuse alopecia: female androgenetic alopecia, telogen effluvium and alopecia areata - part I. An Bras Dermatol. 2012; 87(5):742-47. doi: 10.1590/ S0365-05962012000600010.

16. Kantor J, Kessler LJ, Brooks DG, Cotsarelis G. Decreased serum ferritin is associated with alopecia in women. J Invest Dermatol. 2003;121(5):985-88. doi: 10.7860/JCDR/2015/14089.6170.

17. Burman KD, McKinley-Grant L. Dermatologic aspects of thyroid disease. Clin Dermatol. 2006;24(4):247-55. doi: 10.1016/j. clindermatol.2006.04.010.

18. Freinkel RK, Freinkel N. Hair growth and alopecia in hypothyroidism. Arch Dermatol. 1972;106(3):34952. doi: 10.1001/archderm.1972.01620120037007.

19. Moeinvaziri M, Mansoori P, Holakooee K, Safaee Naraghi Z, Abbasi A. Iron Status in Diffuse Telogen Hair Loss among Women. Acta Dermatovenerol Croat. 2009; 17(4):279-84.

20. Hard S. Non-anemic iron deficiency as an etiologic factor in diffuse loss of hair of the scalp in women. Acta Derm Venereol. 1963; 43:562-9.

21. Rushton DH, Ramsay ID, James KC, Norris MJ, Gilkes JJ. Biochemical and trichological characterization of difuse alopecia in women. $\mathrm{Br} \mathrm{J}$ Dermatol. 1990;123(2):187-97. doi: 10.1111/j.13652133.1990.tb01846.x.

22. Van Neste DJ, Rushton DH. Hair problems in women. Clin Dermatol. 1997;15(1):113-25. doi: 10.1016/S0738-081X(96)00114-9.

23. White MI, Currie J, Williams MP. A study of the tissue iron status of patients with alopecia areata. Br J Dermatol. 1994; 130(2):261-63. doi: 10.1111/ j.1365-2133.1994.tb02917.x.

24. Boffa MJ, Wood P, Griffiths CE. Iron sta.tus of patients with alopecia areata. $\mathrm{Br} \mathrm{J}$ Dermatol. 1995;132(4):662-4. doi: 10.1111/j.1365-2133.1995. tb08727.x. 